\title{
Grating induced transparency (GIT) and the dark mode in optical waveguides
}

\author{
Hsi-Chun Liu and Amnon Yariv \\ Department of Electrical Engineering and Department of Applied Physics, California Institute of Technology \\ Pasadena, California 91125 \\ hliu@caltech.edu
}

\begin{abstract}
We propose and describe a new class of optical modes consisting of superposition of three waveguide modes which can be supported by a few-mode waveguide spatially modulated by two co-spatial gratings. These supermodes bear a close, but not exact, formal analogy to the three-level quantum states involved in EIT and its attendant slow light propagation characteristics. Of particular interest is the supermode which we call the dark mode in which, in analogy with the dark state of EIT, one of the three uncoupled waveguide modes is not excited. This mode has unique dispersion characteristics that translate into a slow light propagation which possesses high bandwidth-delay product and can form the basis for a new generation of optical resonators and lasers.
\end{abstract}

(C)2009 Optical Society of America

OCIS codes: (230.7370) Waveguides; (050.2770) Gratings; (230.5750) Resonators.

\section{References and links}

1. S. E. Harris, "Electromagnetically induced transparency," Phys. Today 50(7), 36-42 (1997).

2. L. V. Hau, S. E. Harris, Z. Dutton, and C. H. Behroozi, "Light speed reduction to 17 metres per second in an ultracold atomic gas," Nature 397(6720), 594-598 (1999).

3. C. Liu, Z. Dutton, C. H. Behroozi, and L. V. Hau, "Observation of coherent optical information storage in an atomic medium using halted light pulses," Nature 409(6819), 490-493 (2001).

4. D. D. Smith, H. Chang, K. A. Fuller, A. T. Rosenberger, and R. W. Boyd, "Coupled-resonator-induced transparency," Phys. Rev. A 69(6), 063804 (2004).

5. L. Maleki, A. B. Matsko, A. A. Savchenkov, and V. S. Ilchenko, "Tunable delay line with interacting whispering-gallery-mode resonators," Opt. Lett. 29(6), 626-628 (2004).

6. M. F. Yanik, W. Suh, Z. Wang, and S. Fan, "Stopping light in a waveguide with an all-optical analog of electromagnetically induced transparency," Phys. Rev. Lett. 93(23), 233903 (2004).

7. A. Naweed, G. Farca, S. I. Shopova, and A. T. Rosenberger, "Induced transparency and absorption in coupled whispering-gallery microresonators," Phys. Rev. A 71(4), 043804 (2005).

8. Q. F. Xu, S. Sandhu, M. L. Povinelli, J. Shakya, S. H. Fan, and M. Lipson, "Experimental realization of an onchip all-optical analogue to electromagnetically induced transparency," Phys. Rev. Lett. 96(12), 123901 (2006).

9. Q. F. Xu, J. Shakya, and M. Lipson, "Direct measurement of tunable optical delays on chip analogue to electromagnetically induced transparency," Opt. Express 14(14), 6463-6468 (2006).

10. K. Totsuka, N. Kobayashi, and M. Tomita, "Slow light in coupled-resonator-induced transparency," Phys. Rev. Lett. 98(21), 213904 (2007).

11. L. Yosef Mario, and M. K. Chin, "Optical buffer with higher delay-bandwidth product in a two-ring system," Opt. Express 16(3), 1796-1807 (2008).

12. Y. F. Xiao, B. K. Min, X. Jiang, C. H. Dong, and L. Yang, "Coupling Whispering-Gallery-Mode Microcavities With Modal Coupling Mechanism,” IEEE J. Quantum Electron. 44(11), 1065-1070 (2008).

13. A. Yariv, and P. Yeh, Photonics: Optical Electronics in Modern Communications (Oxford University Express, New York, 2007).

14. E. Peral, and A. Yariv, "Supermodes of grating-coupled multimode waveguides and application to mode conversion between copropagating modes mediated by backward Bragg scattering," J. Lightwave Technol. 17(5), 942-947 (1999).

15. D. Marcuse, Theory of Dielectric Optical Waveguides (Academic Press, New York and London, 1974).

16. L. I. Schiff, Quantum Mechanics (McGraw-Hill, New York, 1955).

17. X. Sun, H.-C. Liu, and A. Yariv, "Adiabaticity criterion and the shortest adiabatic mode transformer in a coupled-waveguide system," Opt. Lett. 34(3), 280-282 (2009).

\#111538 - \$15.00 USD Received 18 May 2009; revised 21 Jun 2009; accepted 21 Jun 2009; published 26 Jun 2009 


\section{Introduction}

Electromagnetically induced transparency (EIT) is a phenomenon that comes from quantum destructive interference between excitation pathways to the upper level of an atomic threelevel system [1]. The combination of absorption cancellation and strong dispersion has led to the observation of very slow and stored light [2,3]. In these experiments, atoms are prepared in a "dark", coherent superposition of the two lower levels, a stationary eigenstate of the system of a three-level atom and two laser fields.

Classical analogs of EIT can be established in coupled optical resonators, referenced as coupled-resonator-induced transparency (CRIT) [4], where the mode splitting is a classical counterpart of dressed states arising from ac Stark effect. Several configurations of coupled resonators to obtain EIT-like resonances have been proposed and experimentally demonstrated [4-12]. These EIT-like resonances demonstrate much narrower linewidth than those of individual resonators.

In this paper we propose to use a three-mode waveguide modulated by two co-spatial gratings as an optical analog to EIT. The three waveguide modes play the roles of the three quantum states in EIT, while the gratings are counterparts of the electromagnetic waves. Unlike CRIT, where the waveguide-resonator and inter-resonator coupling occur only at discrete points, the coupling of waveguide modes by gratings is continuous along the waveguide. This enables us to describe our system by coupled mode equations, in analogy to the Hamiltonian in the EIT system. The counterpart of the dark state in EIT is one of the supermodes of the waveguide-plus-grating system. By inserting phase shifts or apodizing the gratings, we are able to utilize the slow dark mode and produce EIT-like resonance. The structures can be a new class of optical resonators or optical delay lines.

\section{Grating induced transparency and the Dark mode}

Guided by the formalism of the EIT three quantum state atomic system, we consider a waveguide supporting three distinct spatial modes with complex amplitudes $a, b, c$, propagation constants $\beta_{a}, \beta_{b}, \beta_{c}$, and containing two co-spatial gratings (one connects $a$ and $b$; the other connects $b$ and $c$ ). We do not restrict the direction of propagation of each mode. In the case of weak perturbation, the eigenmodes of the waveguide-plus-grating system, the supermodes, can be taken as linear superpositions of the unperturbed modes $a, b$, $c$, which obey the coupled Eqs. (13):

$$
\begin{aligned}
& \frac{d a}{d z}=-j \beta_{a} a+\kappa_{a b} \exp \left(-j \frac{2 \pi}{\Lambda_{a b}} z\right) b \\
& \frac{d b}{d z}=-j \beta_{b} b+\kappa_{b a} \exp \left(j \frac{2 \pi}{\Lambda_{a b}} z\right) a+\kappa_{b c} \exp \left(j \frac{2 \pi}{\Lambda_{c b}} z\right) c \\
& \frac{d c}{d z}=-j \beta_{c} c+\kappa_{c b} \exp \left(-j \frac{2 \pi}{\Lambda_{c b}} z\right) b
\end{aligned}
$$

where $\Lambda_{a b}=2 \pi /\left(\beta_{a 0}-\beta_{b 0}\right)$ and $\Lambda_{b c}=2 \pi /\left(\beta_{c 0}-\beta_{b 0}\right)$ are the periods of the two gratings designed for a center frequency $\omega_{0} . \kappa_{i j}(i, j=a, b, c)$ are the coupling coefficient connecting modes $i$ and $j$. By introducing new variables $A, B, C$, defined by $a(z)=A(z) \exp \left(-j \beta_{a 0} z\right), b(z)=$ $B(z) \exp \left(-j \beta_{b 0} z\right), c(z)=C(z) \exp \left(-j \beta_{c 0} z\right)$, we arrive at a z-independent coupling matrix:

$$
\frac{d}{d z}\left[\begin{array}{c}
A \\
B \\
C
\end{array}\right]=\left[\begin{array}{ccc}
-j \delta_{a} & \kappa_{a b} & 0 \\
\kappa_{b a} & -j \delta_{b} & \kappa_{b c} \\
0 & \kappa_{c b} & -j \delta_{c}
\end{array}\right] \cdot\left[\begin{array}{c}
A \\
B \\
C
\end{array}\right],
$$

where $\delta_{i} \equiv \beta_{i}-\beta_{i 0}$ is the detuning of the propagation constant. In the neighborhood of $\omega_{0}, \delta_{i}=$ $n_{i}(\Delta \omega) / \mathrm{c}$ where $n_{i}$ is the group index of each mode and $\Delta \omega=\omega-\omega_{0}$. The eigenvectors of the

\#111538 - \$15.00 USD Received 18 May 2009; revised 21 Jun 2009; accepted 21 Jun 2009; published 26 Jun 2009 
matrix are the supermodes of the perturbed waveguide, while the eigenvalues are the propagation constants of the supermodes. For example, the electric field of a supermode $\left[v_{a} v_{b}\right.$ $\left.v_{c}\right]^{\mathrm{T}}$ with a propagation constant $\mu$ is given by $\exp (-i \mu z)\left[v_{a} \exp \left(-i \beta_{a 0} z\right) \quad \boldsymbol{E}_{a}(x, y)+v_{b}\right.$ $\left.\exp \left(-i \beta_{b 0} z\right) \quad \boldsymbol{E}_{b}(x, y)+v_{c} \exp \left(-i \beta_{c 0} z\right) \quad \boldsymbol{E}_{c}(x, y)\right]$, where $\boldsymbol{E}_{i}(x, y)$ is the transverse mode profile. At center frequency $\omega_{0}$, one of the supermodes is proportional to $\left[\kappa_{b c} 0-\kappa_{b a}\right]^{\mathrm{T}}$, with the eigenvalue equal to 0 . In this supermode, which we name the dark mode, the intermediate mode $B$ is unexcited. For comparison, the coupled equation of EIT ignoring decay rates is [1]

$$
\frac{d}{d t}|\Psi\rangle=\left[\begin{array}{ccc}
-j \Delta \omega & -\frac{j}{2} \Omega_{p} & 0 \\
-\frac{j}{2} \Omega_{p} & 0 & -\frac{j}{2} \Omega_{c} \\
0 & -\frac{j}{2} \Omega_{c} & 0
\end{array}\right]|\Psi\rangle,
$$

where $\Omega_{p}$ and $\Omega_{c}$ are the Rabi frequencies of the probe and coupling beams, respectively, and $\Delta \omega$ is the frequency detuning of the probe beam. Equations (2) and (3) are similar on resonance. They are respectively equations of space and time. Conservation of energy defines the relation between $\kappa_{i j}$ and $\kappa_{j i}$ [13]: If mode $i$ and $j$ copropagate, $\kappa_{i j}=-\kappa_{j i}{ }^{*}$; if mode $i$ and $j$ counterpropagate, $\kappa_{i j}=\kappa_{j i}{ }^{*}$. Suppose $A$ is the input forward mode, there are four different choices of propagating directions of mode $B$ and $C$ (Fig. 1). The formal similarity between Eqs. (2) and (3) depends on the nature of the $\kappa_{i j}$ coefficients, which implies both modes $B$ and $C$ are forward propagating (Fig. 1(a)). This makes sense since in quantum mechanics each state evolves forward with time. Nevertheless, the choice which is the most interesting for our slow light phenomenology is one where mode $A$ is forward while $B$ and $C$ are backward propagating (Fig. 1(d)). A short-period grating connects modes A and B $\left(\kappa_{a b}\right)$, while a longperiod grating connects modes $\mathrm{B}$ and $\mathrm{C}\left(\kappa_{b c}\right)$. The coupling constants obey $\kappa_{b c}=-\kappa_{c b} *$ and $\kappa_{a b}$ $=\kappa_{b a} *$. By shifting constant phases of variables $A, B$, and $C$, Eq. (2) can be rewritten as

$$
\frac{d}{d z}\left[\begin{array}{l}
A \\
B \\
C
\end{array}\right]=\left[\begin{array}{ccc}
-j \delta_{a} & \kappa_{a b} & 0 \\
\kappa_{a b} & -j \delta_{b} & j \kappa_{b c} \\
0 & j \kappa_{b c} & -j \delta_{c}
\end{array}\right] \cdot\left[\begin{array}{l}
A \\
B \\
C
\end{array}\right] \equiv \boldsymbol{K} \cdot\left[\begin{array}{l}
A \\
B \\
C
\end{array}\right],
$$

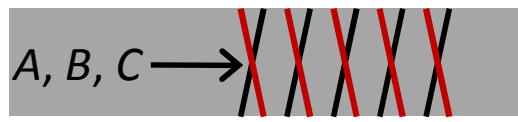

(a)

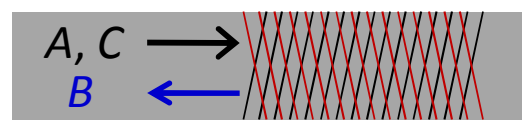

(c)

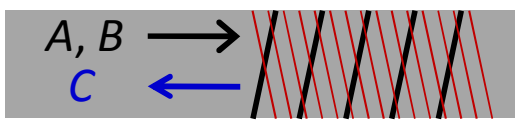

(b)

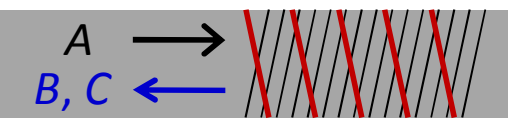

(d)

Fig. 1. Four configurations of the directions of the three modes. The black grating $(/)$ couples modes $A$ and $B$, while the red grating $(\backslash)$ couples mode $B$ and $C$. The gratings are short-period or long-period depending on the directions of the connected modes.

where $\boldsymbol{K}$ is defined as the coupling matrix. Now both $\kappa_{a b}$ and $\kappa_{b c}$ are real numbers. At $\Delta \omega=0$, if $\left|\kappa_{a b}\right| \neq\left|\kappa_{b c}\right|$, the corresponding eigenvectors and eigenvalues are: 
Dark mode:

$$
\begin{aligned}
& \frac{1}{\sqrt{\kappa_{a b}{ }^{2}+\kappa_{b c}^{2}}}\left[\begin{array}{c}
\kappa_{b c} \\
0 \\
j \kappa_{a b}
\end{array}\right], \text { eigenvalue }=0 ; \\
& \propto\left[\begin{array}{c}
-j \kappa_{a b} \\
\pm \sqrt{\kappa_{b c}{ }^{2}-\kappa_{a b}^{2}} \\
\kappa_{b c}
\end{array}\right], \text { eigenvalues }= \pm j \sqrt{\kappa_{b c}{ }^{2}-\kappa_{a b}{ }^{2}} \equiv \pm j \Delta \beta_{0} .(6)
\end{aligned}
$$

the two bright modes

The three eigenvectors are not orthogonal to each other. They are nearly parallel when $\left|\kappa_{a b}\right|$ and $\left|\kappa_{b c}\right|$ are nearly equal. The two bright modes can propagate only if $\left|\kappa_{a b}\right|<\left|\kappa_{b c}\right|$, in which case the propagation constants $\pm \Delta \beta_{0}$ are real. Interestingly, the dark mode is a "propagating" (non-evanescent) supermode consisting of a superposition of two counter-propagating waves, $A$ and $C$, which results in a group velocity that falls between the positive group velocity of the forward wave $A$ and the negative group velocity of the backward wave $C$ and can be derived by perturbation theory as

$$
v_{g, d a r k}=\frac{1-\alpha^{2}}{n_{a}+\alpha^{2} n_{c}} \cdot c,
$$

where the tuning factor $\alpha \equiv \kappa_{\mathrm{ab}} / \kappa_{b c}$. The group velocity is reduced to 0 when $\alpha$ approaches 1 . We focus on the region $|\alpha|<1$ where the group velocity is positive. Plotting the propagation constants of the three supermodes as functions of $\Delta \omega$ results in the band structure shown in Fig. 2 where $\alpha=0.9$. In general the waveguide is not periodic, unless the ratio of $\Lambda_{a b}$ and $\Lambda_{b c}$ is rational. Therefore, the band structure has two sets of Brillouin zones that repeat the dispersion curves periodically. The three modes intersect at $\Delta \omega=0$ and anti-crossing occurs. A narrow transmission band in the center lies between two band gaps. All the three supermodes can propagate within the transmission band, while no forward-propagating mode exists within the two band gaps since the only propagating supermode consists mainly of backward modes $B$ and $C$. The group velocity dispersion (GVD) of the dark mode in the center $(\Delta \omega=0)$ is zero. The group velocity of the two bright modes is $v_{g, b r i g h t}=-\left(1-\alpha^{2}\right) \cdot c / n$ in the assumption that $n_{a} \approx n_{b} \approx n_{c} \approx n$.

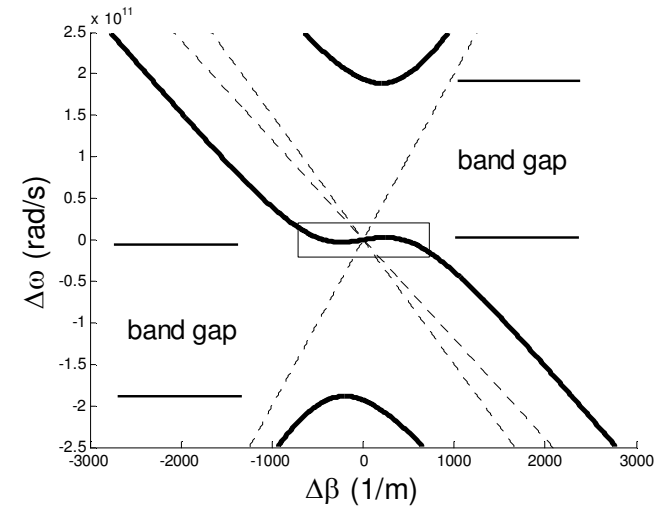

(a)

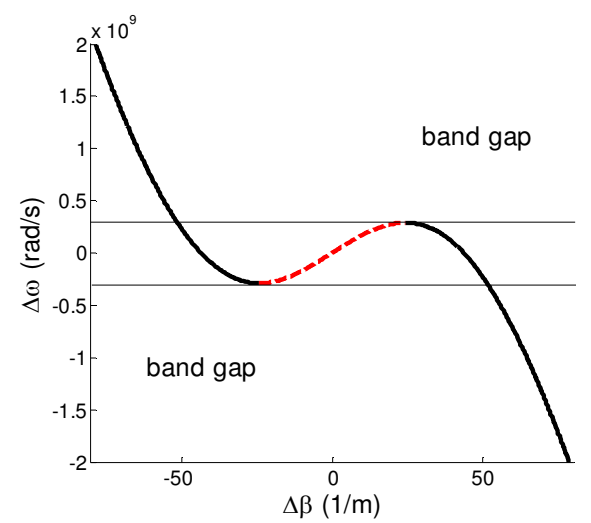

(b)

Fig. 2. (a) Band structures of a GIT waveguide. $n_{a}=1.5, n_{b}=2.5, n_{c}=2 . \kappa_{a b}=90 / \mathrm{m}, \kappa_{b c}=100$ $/ \mathrm{m}$. Dash lines are the band structure without grating perturbation. (b) Zoom-in figure of the bending region. The red dashed curve is the dark mode. 


\section{Uniform GIT waveguide}

To take advantage of the new modes, especially the dark one, we propose two types of structures. The first is a uniform one where both gratings $\left(\kappa_{a b}\right.$ and $\left.\kappa_{b c}\right)$ are of uniform strengths along the length $L$ of the waveguide. If the boundary condition corresponds to a single input mode $A$ at $z=0$ and no input backward modes $B$ and $C$ at $z=L$, the field at $z=L$ is proportional to $\left[\begin{array}{lll}1 & 0 & 0\end{array}\right]^{\mathrm{T}}$, which decomposes into all the three supermodes. Transmission of mode $A$ and field distribution along the structure can be obtained by back propagating the three supermodes to $z=0$. Unity transmission at $\Delta \omega=0$ results if the length is a multiple of

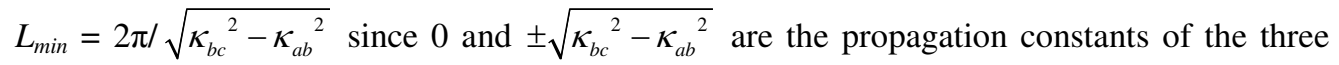
supermodes. Figures 3(a) and 3(b) show the transmission spectrum of a waveguide with $\alpha=$ $0.9, \kappa_{b c}=1,000 / \mathrm{m}$, and $L=L_{\min }=1.44 \mathrm{~cm}$. The transmission spectrum is similar to that of EIT. The FWHM bandwidth is $66.2 \mathrm{MHz}$, and the group delay in the center wavelength is 4.97 ns with group velocity reduction of 71.3 (compared to group velocity of mode $A$ ).

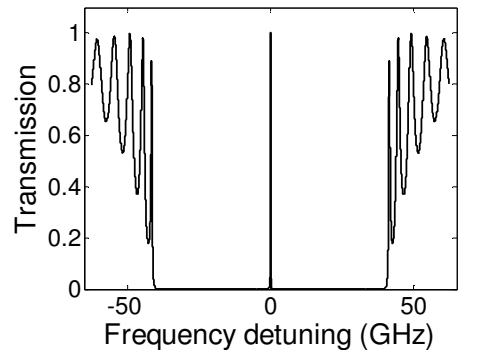

(a)

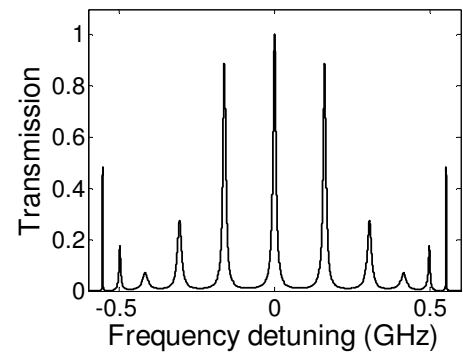

(c)

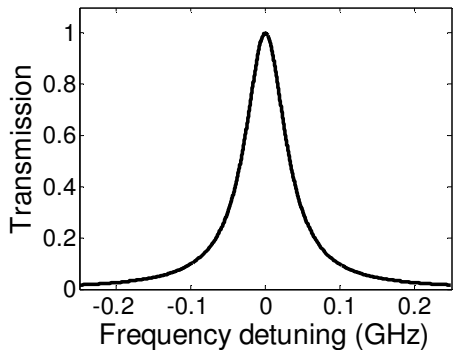

(b)

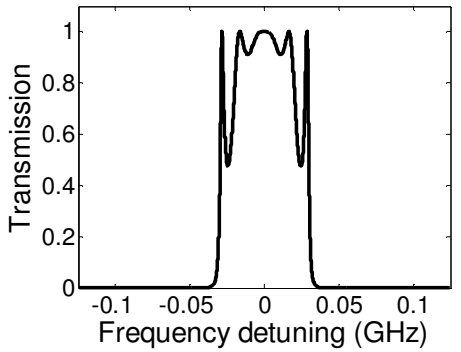

(d)

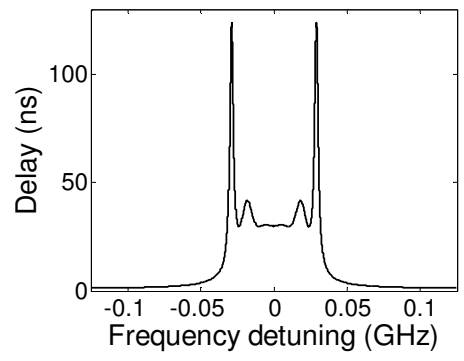

(e)

Fig. 3. (a) Transmission spectrum of a uniform structure. $\kappa_{a b}=900 / \mathrm{m}, \kappa_{b c}=1,000 / \mathrm{m}$, and $L=$ $L_{\min }=1.44 \mathrm{~cm}$. The refractive indices are $n_{a}=1.45, n_{b}=1.425$, and $n_{c}=1.4$. (b) Transmission spectrum in a narrower span of the same structure in (a). (c) Transmission spectrum of a uniform structure with $L=6 L_{\min }$. (d) Transmission spectrum of a uniform structure with $L=$ $6 L_{m i n}$ and periodic inversion of $\kappa_{a b}$. (e) Group delay of the structure in (d). 
The case of uniform gratings occupying $0<z<L$ can be viewed as a resonator where the two backward bright modes are reflected into the forward dark mode at $z=0$ and conversely at $z=L$. The round-trip phase of the cavity includes the forward propagation of the dark mode, the backward propagation of the two bright modes, and the phase of reflectance of the two mirrors. This explains why $L$ has to be a multiple of $L_{\min }$ for resonance at $\Delta \omega=0$. Since the transmission of mode $A$ depends on phase matching of the three supermodes, the bandwidth is extremely narrow and the group velocity reduction is much larger than that of the dark mode in Eq. (7). At $\Delta \omega=0$, the field distributions of the individual modes are

$$
\begin{aligned}
& A(z)=\frac{1}{1-\alpha^{2}}\left[1-\alpha^{2} \cos \left(\Delta \beta_{0} z\right)\right] \\
& B(z)=\frac{\alpha}{\sqrt{1-\alpha^{2}}} \sin \left(\Delta \beta_{0} z\right) \\
& C(z)=\frac{j \alpha}{1-\alpha^{2}}\left[1-\cos \left(\Delta \beta_{0} z\right)\right]
\end{aligned}
$$

assuming the input $A(0)=1$. Figure 4 plots the field distribution for $\alpha=0.95$ and $L=2 L_{\text {min }}$. Since the three supermodes are nearly parallel, they interfere constructively to extremely high intensities of individual modes $A, B, C$ in the middle of the structure, and destructively interfere to small intensity of $A$ at the two ends. The quality factor $Q$ of the resonator defined as $\omega \cdot$ (energy stored) / (power loss) can be obtained from Eq. (8) assuming $n_{a} \approx n_{b} \approx n_{c}$ and is given by

$$
Q=\beta_{a} L \frac{1+2 \alpha^{2}}{\left(1-\alpha^{2}\right)^{2}},
$$

where $L$ is fixed to be a multiple of $L_{\min }$. If $\alpha$ is close to 1 , say 0.999 for example, the factor (1 $\left.+2 \alpha^{2}\right) /\left(1-\alpha^{2}\right)^{2}$ is as high as $7.5 \times 10^{5}$, while the length required is $14 \mathrm{~cm}$ assuming $\kappa_{b c}=$ $1,000 / \mathrm{m}$. By employing gain in the structure with a high quality factor we can make a laser with the minimum threshold gain occurring when $L$ is a multiple of $L_{\min }$ and is inversely proportional to the quality factor.

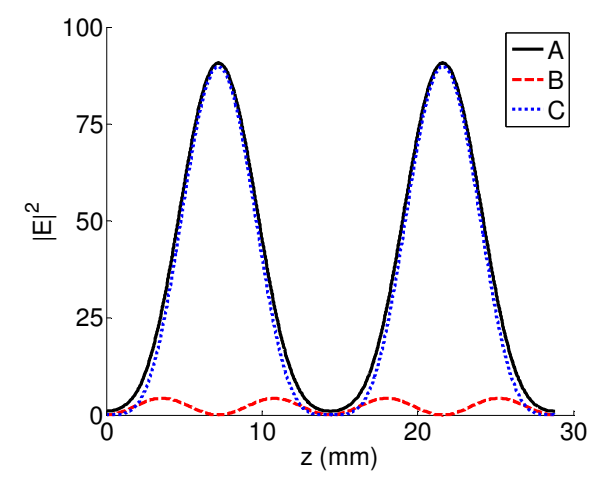

Fig. 4. Energy distribution of a uniform structure with $L=2 L_{\min }$ and an input $A(0)=1 . \kappa_{a b}=$ $900 / \mathrm{m}, \kappa_{b c}=1,000 / \mathrm{m}$, and $L=2.88 \mathrm{~cm}$. The refractive indices are $n_{a}=1.45, n_{b}=1.425$, and $n_{c}=1.4$.

For the example in Figs. 3(a) and 3(b), the phase shift across the transmission band is about $\pi$, limiting the delay-bandwidth product less than 0.5 , like a single resonator. Cascading $N$ resonators, namely increasing $L$ to $N \cdot L_{\text {min }}$, will result in more delay but reduced bandwidth, since the phase mismatch of the three supermodes is approximately proportional to $(\Delta \omega) L$. In fact, as shown in Fig. 3(c), there are $2 N-1$ peaks in the transmission spectrum, each with a phase shift of $\pi$ across, limiting the delay-bandwidth product still less than 0.5 . To break this 
limit, we propose to invert the sign of $\alpha\left(=\kappa_{a b} / \kappa_{b c}\right)$ every $L_{\min }$ along the waveguide, as illustrated in Fig. 5(a). By doing this periodic inversion, it can be shown that the first-order term of the reflectance at $z=0$ as a function of $\Delta \omega$ is cancelled out when $N$ is even. Figures 3(d) and 3(e) show the transmission spectrum and group delay of the modified waveguide with $L=N \cdot L_{\min }$, where the bandwidth is not reduced and the envelope of the ripples is approximately a quadratic function. The group delay at $\Delta \omega=0$ and the quality factor of the modified waveguide is exactly the same as the original one, while the delay-bandwidth product is unlimited and the total phase shift across the bandwidth is $N \pi$.

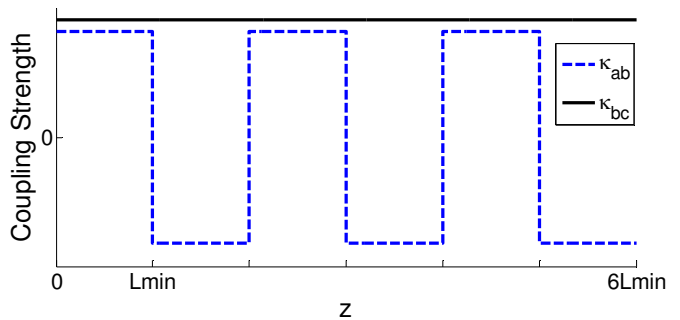

(a)

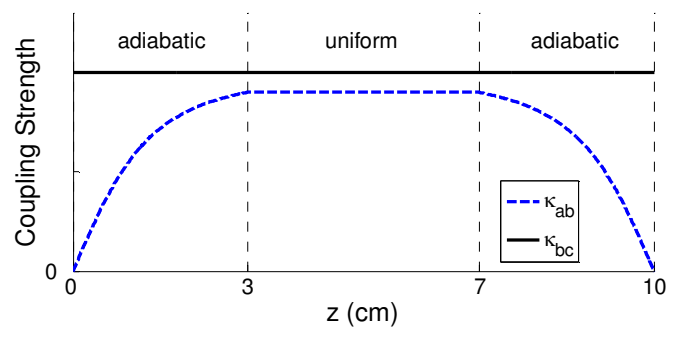

(b)

Fig. 5. (a) $\kappa_{a b}$ and $\kappa_{b c}$ in a uniform structure with periodic inversion. (b) $\kappa_{a b}$ and $\kappa_{b c}$ in an adiabatic structure. $L_{a d}$ and $L_{u n i}$ are the lengths of the adiabatic and uniform region, respectively.

\section{Adiabatic GIT waveguide}

The second structure limiting the excitation to the dark mode alone is achieved by employing adiabatic transition $[14,15]$. This is accomplished by changing the structure (waveguide + gratings) sufficiently slowly along the direction of propagation $(z)$ so that the propagating wave remains in the same local eigenmode. As shown in Fig. 5(b), the waveguide consists of two adiabatic regions at the two ends and a uniform region in the middle. $\kappa_{b c}$ is set to be a constant throughout the structure, while $\kappa_{a b}$, and thus $\alpha$, are functions of $z$. The input mode $A$ entering the structure encounters only grating $\kappa_{b c}$ and is thus the dark mode (Eq. (5)) where it will remain till exiting the structure. As it propagates, the grating $\kappa_{a b}$ is turned on spatially adiabatically. In the middle of the structure, the dark mode which now has a mode $C$ component possesses a small group velocity. At the output, $\kappa_{a b}$ is adiabatically decreased to 0 , and the propagating wave is transformed back, always staying dark, to mode $A$ at the exit.

The adiabatic condition ensuring that a wave remains in the same (dark) mode is defined as the situation where the fraction of the energy converted to other modes never exceeds an arbitrarily chosen small number $\varepsilon$. The adiabatic condition of the GIT waveguide and an optimal function $\alpha(z)$ can be derived in a similar way as in quantum mechanics [16] and an adiabatic mode converter [17], with careful treatment of the non-orthogonal basis. The three eigenvectors in Eqs. (5) and (6) should be renormalized such that the total forward energy flow is 1 or -1 , given by 


$$
v_{\text {dark }}=\frac{1}{\sqrt{1-\alpha^{2}}}\left[\begin{array}{c}
1 \\
0 \\
j \alpha
\end{array}\right], v_{b r i g h t-1,2}=\frac{1}{\sqrt{2\left(1-\alpha^{2}\right)}}\left[\begin{array}{c}
-j \alpha \\
\pm \sqrt{1-\alpha^{2}} \\
1
\end{array}\right] .
$$

When decomposing a vector $\boldsymbol{x}=[A B C]^{\mathrm{T}}$ into the three supermodes, the amplitude of each supermode can be determined by the product with the associated left eigenvectors of the coupling matrix $\boldsymbol{K}$ since $\boldsymbol{u}_{i}^{T} \boldsymbol{v}_{j}=\delta_{i j}$, where $\boldsymbol{u}_{i}{ }^{T}$ and $\boldsymbol{v}_{i}$ are respectively the $i$-th left and right eigenvectors associated with the eigenvalue $\lambda_{i}$ of the coupling matrix $\boldsymbol{K}$. The fields $\boldsymbol{x}$ can be written as the superposition of the supermodes $v_{i}$,

$$
\boldsymbol{x}(z)=\sum_{i=d a r k, b r i g h t} a_{i}(z) \boldsymbol{v}_{i}(z) \exp \left[-j \int_{0}^{z} \beta_{i}\left(z^{\prime}\right) d z^{\prime}\right] .
$$

Substituting Eq. (11) into the coupled equations $\frac{d}{d z} \boldsymbol{x}(z)=\boldsymbol{K}(z) \boldsymbol{x}(z)$ (Eq. (4)) leads to

$$
\sum_{i=d a r k, b r i g h t}\left[\left(\partial_{z} a_{i}\right) \boldsymbol{v}_{i}+a_{i}\left(\partial_{z} \boldsymbol{v}_{i}\right)\right] \exp \left[-j \int_{0}^{z} \beta_{i}\left(z^{\prime}\right) d z^{\prime}\right]=0 .
$$

For adiabatic transition of the Dark mode $\left(v_{\text {dark }}\right), a_{\text {dark }} \approx 1$ and $a_{\text {bright }-1,2} \approx 0$. Multiplying $\boldsymbol{u}_{\text {bright-1 }}{ }^{\mathrm{T}}$ from the left side results in

$$
d a_{\text {bright-1}} / d z=\boldsymbol{u}_{\text {bright }-1}^{T}\left(\partial_{z} \boldsymbol{v}_{\text {dark }}\right) \exp \left[\int_{0}^{z} \beta_{\text {bright-1 }}\left(z^{\prime}\right) d z^{\prime}\right],
$$

since $\beta_{\text {dark }}=0 . \boldsymbol{u}_{\text {bright }-1}{ }^{T}\left(\partial_{z} v_{\text {dark }}\right)$ can be shown to be $\frac{j}{\sqrt{2}\left(1-\alpha^{2}\right)} \frac{\partial \alpha}{\partial z}$, and with the assumption that it is slowly varying compared to the exponential term, Eq. (13) can be solved as

$$
a_{\text {bright-1 }}(z)=\frac{-\sqrt{2} j}{\kappa_{b c}\left(1-\alpha^{2}\right)^{3 / 2}} \frac{\partial \alpha}{\partial z} \sin \left(\frac{\beta_{\text {bright-1 }}}{2} z\right) \exp \left[j \frac{\beta_{\text {bright-1 }}}{2} z\right]
$$

Likewise for $a_{\text {bright-2 }}(z)$. The adiabatic condition where only the Dark mode exists requires $\left|a_{\text {bright-1 }}(z)\right|^{2}+\left|a_{\text {bright }-2}(z)\right|^{2} \leq \varepsilon$, which gives

$$
\frac{d \alpha}{d z} \leq \frac{\kappa_{b c}}{4}\left(1-\alpha^{2}\right)^{\frac{3}{2}} \sqrt{\varepsilon}
$$

The closer $\alpha$ is to 1 , the smaller $d \alpha / d z$ is required to achieve adiabaticity. By solving Eq. (15) with equality, we obtain an optimal function $\alpha(z)$ given by

$$
\alpha(z)=\sin ^{-1}(\tan (a z)),
$$

where $a$ is a scaling constant. It takes infinite length to transform $\alpha$ to 1 to reach zero group velocity. Figure 6 shows the transmission spectrum and group delay of an adiabatic GIT structure, where $\alpha$ of the uniform region is 0.9 and the total length is $10 \mathrm{~cm}$. The transmission spectrum resembles a rectangular function, with a bandwidth of $4.48 \mathrm{GHz}$, equal to that of the dark mode of the uniform region. The group delay mainly depends on the group velocity of the dark mode of the uniform region and is $2.92 \mathrm{~ns}$ at the center wavelength with group velocity reduction of 6.04. The delay-bandwidth product, which is 13.1 in this case, can be made arbitrarily large by increasing the length of the uniform region without reducing the bandwidth. 


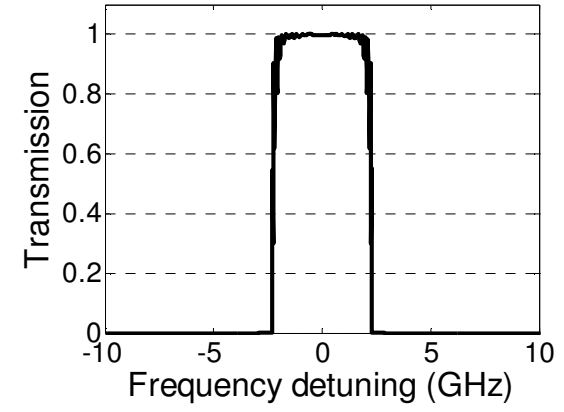

(a)

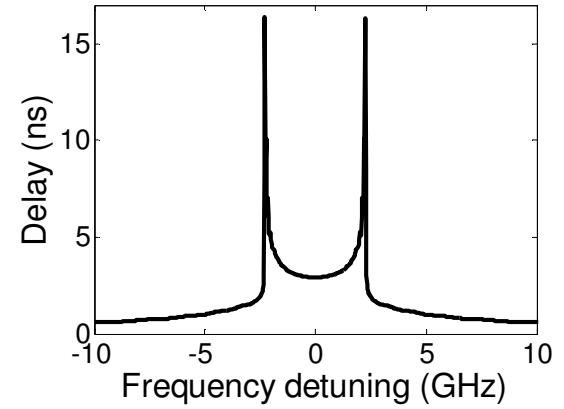

(b)

Fig. 6. (a) Transmission and (b) group delay of an adiabatic structure. $L_{a d}=3 \mathrm{~cm}, L_{u n i}=4 \mathrm{~cm}$, $\kappa_{b c}=4,000 / \mathrm{m}$, and $\alpha_{\max }=0.9$. The refractive indices are $n_{a}=1.45, n_{b}=1.425$, and $n_{c}=1.4$.

\section{Discussion and conclusion}

We have analyzed the EIT-analog waveguide and proposed two types of structures to control the propagation of supermodes. This GIT waveguide can be implemented using a few-mode fiber or a few-mode on-chip waveguide. One short-period grating and another long-period grating are imposed on the waveguide. For a waveguide with geometric symmetry, the modes are either symmetric or anti-symmetric, so are the gratings that couple the modes. Therefore, the two gratings can be independent of each other if they have opposite symmetry. The symmetry of a grating can be controlled by tilting the grating in fibers or designing the transverse profile of a waveguide grating. The phase indices of the three modes should be carefully chosen, in case any unwanted mode is coupled by one of the gratings, since there are three forward and three backward modes in total. Moreover, to reduce loss, coupling into the radiation modes should be prevented.

In conclusion, the new energy storage mechanism resulting from the internal bouncing of waves between two Bragg gratings gives rise to a new class of propagating modes which includes a dark mode with "slow light" characteristics. These modes can potentially form the basis of a new class of narrow band filters, high Q resonators and lasers. The adiabatic GIT structures which excite the dark mode alone possess high delay-bandwidth product.

\section{Acknowledgements}

The authors acknowledge the support of Taiwan Merit Scholarship TMS-094-2-A-039.

\#111538 - \$15.00 USD Received 18 May 2009; revised 21 Jun 2009; accepted 21 Jun 2009; published 26 Jun 2009 